DOI: $10.5216 /$ rpp.v14i2.12932

\title{
COMPRENDIENDO EL ABANDONO DEL DEPORTE DE LAS CHICAS ADOLESCENTES
}

\author{
Emilia Fernández García \\ Universidad Complutense de Madrid, Madrid, Espanha
}

Resumen: El propósito del trabajo es analizar el abandono deportivo de las chicas adolescentes, identificando sus experiencias y los factores que han contribuido a su retirada de esta práctica.El diseño es de tipo etnográfico e interpretativo, empleándose la entrevista retrospectiva con chicas adolescentes (14-17 años) que han abandonado la práctica deportiva recientemente. El análisis cualitativo de los datos se ha realizado con el programa "Nud*ist Vivo" versión 6.0. Los resultados muestran la interacción de un conjunto de factores de influencia entre los que destacan la dedicación académica y las interacciones sociales, que se configuran como base de la continuidad, principalmente la influencia ejercida por la familia y las interacciones personales como determinantes del ambiente deportivo.

Palabras clave: Abandono. Deporte. Actividad Física. Chicas Adolescentes.

\section{Introducción}

El hecho de que la participación de los jóvenes en la práctica del deporte y de la actividad física descienda en los años de la adolescencia, y que éste fenómeno resulte mucho más acusado en las chicas que en los chicos (MARTÍN, 1997; CURRIE, ROBERTS, MORGAN et al, 2004; CURRIE, GABHAINN, GODEAU, et al., 2008; TELAMA, NAUL, NUPPONEN et al, 2002; CORBIN, PANGRAZI Y LE MEASURIER, 2004; SERRA, 2006; FERNÁNDEZ, VÁZQUEZ, CAMACHO et al, 2006), constituye un tema de interés, desde una perspectiva de género, ya que, lo que en definitiva supone para las chicas es el mantenimiento de una desigualdad "real" en los beneficios e influencias positivas constatadas que la participación deportiva proporciona.

Sin embargo, y aunque en la literatura existe una trayectoria de estudio sobre el fenómeno del abandono deportivo (GOULD, 2007), éste ha sido analizado principalmente en jóvenes deportistas con alta implicación deportiva, integrándose en los análisis no sólo la retirada de toda actividad sino también el abandono de una actividad para ser sustituida por otra, pero en menor medida se han centrado los trabajos en una población adolescente general, cuya implicación con esta actividad puede enmarcarse principalmente dentro de un deporte de base, con propósitos mas recreativos y de socialización que de desarrollo deportivo personal hacia altos niveles de excelencia. Adicionalmente, todavía es necesario un mayor cuerpo de conocimiento y estudios particularmente centrados en las chicas, ya que aún se conoce poco sobre sus puntos de vista en relación con el ámbito del deporte (SLEAP y WORMALD, 2001). La respuesta a la pregunta de ¿por qué se produce el abandono de las chicas en el deporte? debe buscarse analizando un conjunto de mediadores $\mathrm{y}$ factores de influencia, e interpretando aquellas circunstancias que afectan más, y especialmente, a las chicas. 
En este sentido, algunos trabajos dirigidos específicamente a las chicas adolescentes recogen un conjunto de factores que parecen contribuir a la retirada de las jóvenes de su actividad físico-deportiva. Así, desde las iniciales contribuciones de White y Coakley (1986) se identifican como influencia las percepciones de menor competencia o el menor apoyo social recibido por las chicas, así como los conflictos de intereses, hasta las razones de falta de tiempo, de éxito o de diversión constatadas por Gould (1987), o las actitudes previas negativas o de indiferencia configuradas a partir de la participación de las adolescentes en las clases de Educación Física (SAARINEN, 1987). Adicionalmente, se constata también que la realización de otras actividades, muchas de ellas académicas, es uno de los motivos principales para no practicar actividades deportivas, siendo aquí los padres el principal indicador de orientación de las jóvenes para la participación de sus hijos e hijas en deportes organizados (RODRÍGUEZ, 1998).

En los últimos años, la indagación sobre los factores que afectan específicamente a las chicas se ha llevado también a cabo a partir de metodologías cualitativas e interpretativas que ahondan en las experiencias y vivencias de las propias adolescentes. En esta línea, el trabajo de Dwyer et al (2006), a través de grupos de discusión, identificó como dificultades para la participación de las chicas canadienses un conjunto de factores centrados en el cuerpo y vinculados con la apariencia física y los estereotipos de feminidad, así como la competición, la falta de tiempo relacionada con el tiempo empleado por las jóvenes en el uso de las actuales tecnologías, o la influencia del grupo de amigas, padres y profesorado. Por otra parte, el trabajo de Whitehead y Biddle (2008) realizado con chicas inactivas británicas, reveló la influencia de la percepción de feminidad y de los estereotipos de género como un conflicto vivido por aquellas chicas que mantenían una identidad de género más "femenina", ya que rechazaban las manifestaciones del ejercicio relacionadas con el esfuerzo físico, la sudoración, etc., que las alejaban de su imagen de feminidad. En esta misma línea, y a través de grupos de discusión con chicas adolescentes entre 13 y 15 años, Slater y Tiggemann (2010), identifican el conjunto de razones específicas de género por las que cesó la participación de las chicas en la actividad física y el deporte, delimitándose principalmente como la falta de tiempo, de competencia o de interés, la escasa disponibilidad de actividades, las pobres relaciones de equipo, conflictos con el entrenador o entrenadora y la influencia de los amigos o de la familia.

El conjunto de estos y otros posibles factores, determina de hecho que las chicas adolescentes se vean más afectadas que los chicos en la culminación de su relación con la actividad física-deportiva. Por ello, el presente trabajo indaga en la particular situación que afecta a las chicas adolescentes en su retirada de la actividad física y el deporte, desde un enfoque psico-social que integra el conjunto de experiencias y vivencias de las chicas dentro de un marco de relación e interacción con su entorno. Su propósito final es el de identificar las experiencias vitales de estas chicas en sus relaciones con el deporte, permitiendo una mayor comprensión sobre los factores que han contribuido al abandono de su práctica en edades tempranas. En tanto que éstos factores son percibidos de una manera particular por las jóvenes, es relevante profundizar en la representación que posean las propias chicas sobre el conjunto de circunstancias desencadenantes de su decisión de retirarse de la práctica deportiva.

Pensar a Prática, Goiânia, v. 14, n. 2, p. 1-15, maio/ago. 2011 


\section{Método}

El diseño de la investigación es de carácter etnográfico e interpretativo y centrado en las mujeres adolescentes. Dada la naturaleza de la información que se precisaba recoger (experiencias, opiniones y valoraciones de las chicas adolescentes estudiadas), se utilizó como técnica la entrevista en profundidad con enfoque retrospectivo.

Las participantes son mujeres adolescentes. Se seleccionaron diez estudiantes, de $2^{\circ}$ Ciclo de E.S.O. y Bachillerato, que habían abandonado la práctica deportiva organizada. Del total de las chicas que manifestaron previamente su conformidad para la colaboración, los criterios integrados para realizar la selección de las participantes han sido los siguientes:

- edad, entre 14 y 17 años;

- abandono reciente, dentro de los dos últimos años, de la práctica deportiva;

- diversidad en el tipo de deporte practicado, atendiendo tanto a deportes individuales como colectivos así como su identificación dentro de una tipificación de género como práctica deportiva "femenina", "masculina" o "neutra"; y

- situación académica según su escolarización en centros públicos o privados.

En la tabla 1 se muestran las características que corresponden a cada una de las entrevistadas.

\begin{tabular}{|l|l|l|l|l|}
\hline \multicolumn{5}{|c|}{ Tabla 1. Identificación de las participantes en las entrevistas } \\
\hline Sujetos & $\begin{array}{c}\text { Tipo de } \\
\text { Centro }\end{array}$ & \multicolumn{1}{|c|}{ Deporte practicado } & Edad & \multicolumn{1}{c|}{ Estudios } \\
\hline CR & Privado & Individual (Gimnasia Rítmica) & 16 & 2 ciclo ESO \\
\hline EV & Público & Individual (Gimnasia Deportiva) & 16 & Bachillerato \\
\hline IS & Privado & Individual (Natación) & 15 & 2 ciclo ESO \\
\hline JO & Público & Colectivo (Futbol Sala) & 14 & 2 ciclo ESO \\
\hline NO & Público & Individual (Gimnasia Rítmica) & 16 & Bachillerato \\
\hline PA & Privado & Individual (Atletismo) & 16 & Bachillerato \\
\hline PAT & Público & Colectivo (Futbol Sala) & 16 & 2 ciclo ESO \\
\hline PI & Público & Individual (Tenis) & 17 & 2 ciclo ESO \\
\hline RU & Privado & Individual (Gimnasia Rítmica) & 17 & Bachillerato \\
\hline TA & Público & Colectivo (Voleibol) & 16 & 2 ciclo ESO \\
\hline
\end{tabular}

Procedimiento. Para llevar a cabo la entrevista, se contactó previamente con ocho centros docentes. Previa conformidad y establecimiento de la colaboración con el centro, se remitió a las adolescentes de $2^{\circ}$ ciclo de E.S.O. un protocolo informativo de la investigación, solicitándoles su conformidad en la participación que incluía la aceptación familiar. Entre todas las respuestas afirmativas recogidas, se seleccionaron las participantes a través de la aplicación de los criterios anteriormente expuestos.

Posteriormente, la entrevistadora, con experiencia en dicha técnica y familiarizada previamente con los propósitos de la investigación, contactó personalmente con cada participante, acordando día y hora para la celebración individual de la entrevista, que se 
celebró en dependencias facilitadas por los centros docentes. La entrevista fue grabada, previa aceptación de la entrevistada, y posteriormente transcrita a soporte informático.

Análisis de los datos. Se realizó un tratamiento de análisis de datos cualitativo ${ }^{1}$ (Rodríguez, Gil, García y Etxeberría, J., 1995), a partir de la aplicación del programa "Nud*ist Vivo" versión 6.0. El "sistema de indización" facilitó definir y correlacionar conceptos y categorías, al tiempo que ha permitido la creación y comprobación de nuevas categorías emergentes de los resultados obtenidos mediante la comparación, relación e intersección de las categorías definidas dentro del árbol de indexación. Las interpretaciones que surgieron del proceso de lectura y de análisis se registraron en ficheros asociados a una categoría o a una unidad de texto en particular. La relación de cada unidad de texto con las restantes, así como su contenido textual, permitió examinar las relaciones existentes entre categorías y establecer relaciones de jerarquía entre ellas.

\section{Resultados}

El árbol de indización elaborado para realizar el análisis de los datos cualitativos, parte del nudo clave que se ha denominado "Abandono del deporte". Los espacios o marcos de referencia que constituyen la columna vertebral del análisis, siguiendo la organización que proporcionan las categorías emergentes, se centran en los siguientes: La falta de tiempo; causas escolares: el éxito y el fracaso escolar; la familia en apoyo a la "escuela" y el ambiente deportivo: interacción entre personas.

La falta de tiempo

Si bien en algún caso el abandono deportivo ha tenido razones muy específicas, que se tratan posteriormente, el tiempo se plantea siempre, y en todos los casos, como un factor determinante. Para algunas chicas la falta de tiempo es algo que puede controlarse, siendo otras causas las que provocan el abandono, pero en otras muchas situaciones es la causa que directamente las conduce a dejar de practicar. Las razones, como se expondrá, podrán ser variadas (unas con mayor fuerza que otras), pero todas mantienen en común la falta de tiempo. Tiempo para compaginar el deporte con el estudio, con los amigos, con otros gustos personales.

CR.- [...] yo creo que según la persona, porque si tienen una capacidad más grande por ejemplo que la mía de estudiar, porque yo tengo que machacar mucho las cosas y poder estudiar en menos tiempo las mismas cosas, pues si lo pueden compaginar, pero a una persona que le cueste yo creo que si que es una cosa que le haga dejarlo. (Entrevista a CR. 77)

Puesto que el tiempo es algo que aparece siempre y en el discurso de todas las jóvenes, la interacción con otras circunstancias en las que emplear el tiempo es

1Cuando hablamos de análisis de datos cualitativos, en cualquier caso, nos referimos a tratamientos de los datos que se llevan a cabo generalmente preservando su naturaleza textual, poniendo en práctica tareas de categorización y sin recurrir a las técnicas estadísticas. (Rodríguez, Gil, García y Etxeberría, 1995:201)

Pensar a Prática, Goiânia, v. 14, n. 2, p. 1-15, maio/ago. 2011 
determinante en el abandono, como las causas escolares, que se ha manifestado como un factor de gran importancia para la mayoría de las chicas.

Causas escolares. El éxito y el fracaso escolar.

Para analizar esta cuestión, es necesario indagar el esquema que sigue el principio de utilidad en las entrevistadas, qué aspectos son los que resultan predominantes para que lleguen a retirarse de la práctica deportiva, ya que, puesto que ésta se ha abandonado, para estas chicas existen valores por encima de su ejercicio. Así, nos encontramos, dentro de un planteamiento inicial de confluencia de causas, con la interacción, al parecer negativa, que se produce entre los estudios y la participación en la actividad física, como razón fundamental por la que se justifica el haber abandonado la práctica deportiva.

P.- Bueno ¿ y cual fue el principal motivo de dejarlo?

CR.- Pues fue según vas haciendo cursos, como son más difíciles, pues los estudios hacen que lo dejes, porque te ponen muchos deberes, te hacen estudiar muchas cosas y eso, y luego ya no es lo mismo porque te vas haciendo mayor y hay mas rivalidad entre tus compañeras, siempre empiezas tan bien y luego ya, yo lo hago mejor, tu lo haces peor, yo gano, tu pierdes, y al final no. (Entrevista a CR. 54-59)

P.-¿Y cuáles fueron los principales motivos de abandonar la natación?.

IS.-Lo dejé hace un año y fue por los estudios, por la cantidad de deberes y de exámenes y de estudios que te ponían, pues era exagerado, luego es que también en clase, mi tutora me llegó a decir que como no dejase la natación me suspendía, que repetía curso.

P.-¿Por qué? „¿Porque llevabas mal el curso?.

IS.-Porque había días que, como mandaban tantas cosas, yo llevaba algunas cosas sin hacer a clase y eso todos los profesores se lo dicen al tutor y el tutor te dice a ti. Digo" es que yo voy a natación y no tengo mucho tiempo para estudiar y deberes y antes estudio que los deberes y me dijo: pues o dejas la natación o suspendes el curso". (Entrevista a IS. 67-76)

P.- ¿Y por qué has dejado de hacer gimnasia artística?.

NO.- Porque no tenía tiempo, porque el año pasado empecé a ir a clases particulares de matemáticas por que necesitaba apoyo y eso, y en cuanto salía de las clases particulares tenía que ir a los entrenamientos y ya no me daba tiempo para estudiar, y entonces o dejaba una cosa o dejaba la otra, entonces vi mas necesario dejar el deporte para poder seguir estudiando.

P.- ¿Y ese es el único motivo, o hay algunos más?.

NO.- Fue el principal, los estudios. (Entrevista a NO. 49-52)

Pensar a Prática, Goiânia, v. 14, n. 2, p. 1-15, maio/ago. 2011 
La priorización de valores queda patente en las entrevistadas pues se plantea como fundamental el estudio para obtener una serie de objetivos en el futuro. Esos objetivos guían un planteamiento de utilidad, es decir, servirán a la persona para algo mucho más importante en la sociedad en la que vivimos.

Lo que se persigue por las adolescentes es la posibilidad de obtener un puesto de trabajo, la futura inserción laboral. En definitiva, el planteamiento final es sobre un proyecto de vida: el conseguir un trabajo implicaría la posibilidad de establecer una familia (si se desea), una autonomía económica, unas relaciones con el entorno... algo que se halla para las chicas en una escala más alta que la propia práctica deportiva. Al final, ello llega esto a ser asumido por estas jóvenes.

PI.- Siempre me han dicho que si destacas en el deporte mucho, pues sí sería una pena dejarlo, pero principalmente el estudio es lo que de mayor me va a dar de comer ¿no?, entonces no me han dicho que lo deje ni me han presionado, me han dado libertad total par elegir ¿no?, pero no sé, yo sentía que era lo que tenía que hacer ¿no? (Entrevista a PI. 54)

En cualquier caso, se produce una construcción teórica alrededor del abandono por causas escolares que justifica la decisión. Esas teorías no sólo abarcan a la propia persona sino que se realizan en términos generales.

P.- Muy bien, ¿tu crees que esos motivos son generalizados a otras chicas de tu edad que abandonan el deporte?

PI.- Si, yo pienso que generalmente se da mas eso de que una chica abandone el deporte por estudiar y por prepararse que un chico, es algo que se da bastante mas. (Entrevista a PI. 55-56)

No sólo se han retirado de la práctica del deporte por razones académicas, sino que argumentan esa decisión como algo natural, algo que es decidido por la gran mayoría de personas que han abandonado ésta. Con ello se está normalizando su situación, impidiendo que existan remordimientos y sentimientos de culpa por haber realizado una hipotética decisión incorrecta. Adicionalmente, este refugio en un hecho "generalizable" se identifica por las propias chicas como comportamiento más femenino que masculino.

En principio, puede resultar coherente que, si hay dificultades para compaginar deporte-estudio, los problemas que se presenten en la escuela sean un detonante para abandonar definitivamente la práctica deportiva. Pero no es así; también las chicas que presentan éxito en sus estudios tienen un reforzamiento por continuar por el camino académico, siendo lo accesorio su participación en el deporte, y sufren similar presión para el abandono. Veamos un ejemplo:

P.- Entonces, ¿cuando te empezaste a plantear que tenias que dejarlo?

CR.- Pues en primero de la E.S.O, ya cambia mucho en el colegio, notas el cambio y notas que te cuesta seguir pero luego ya segundo,

Pensar a Prática, Goiânia, v. 14, n. 2, p. 1-15, maio/ago. 2011 
y ya fue en tercero cuando estuve el primer trimestre en gimnasia pero ya no pude y dije: "Aquí ya no puedo más"

P.- Pero ¿qué fue lo que te llevó a decidirte?

CR.- Es que no sé, es eso, es que llegaba ahora mismo las nueve y media de la noche, llegaba la gimnasia y tenias que pensar tengo que ponerme ahora a estudiar y estar mucho tiempo, y era muy tarde a la hora que te acostabas, y luego levantarte pronto porque empezábamos a entrar a las 8 y dije no puedo, porque terminas la semana totalmente rendida. (Entrevista a CR. 64-69)

Existe por tanto una gran presión por el estudio, la cuál se incrementa con cada año que pasa, haciendo poco sostenible una práctica deportiva cuando esta supone cierto nivel de dedicación. Paralelamente, el éxito escolar que obtienen les confirma que éste es una vía válida y segura para obtener un futuro laboral, económico y social. La escala de valores que han ido obteniendo durante toda su vida se reafirma. Deben decidir ante la presión que se está ejerciendo y la fortaleza que progresivamente se ha adquirido en los valores socialmente considerados "más útiles" ofrece pocas alternativas a la decisión de futuro, produciéndose el abandono del deporte.

La familia en apoyo a la "escuela"

En el caso de las jóvenes entrevistadas también se observa su proceso de socialización a partir de la familia. En este caso la familia tiene una gran influencia para determinar el sistema de preferencias. La decisión de abandono deportivo como elección ante "los estudios" es posible en personas que tienen una perspectiva de futuro muy avanzada, pero en una edad temprana, donde estos valores quedan muy alejados para las niñas, es difícil que surja espontáneamente, siendo la propia familia, en gran parte de las ocasiones, la que sirve de guía para esas expectativas.

PI.- Claro, a mí me gustaba y un entrenador de otro equipo habló conmigo y me dijo que era buena e intente meterme, pero ya empecé el curso otra vez y me dijo mi madre que a ver si no lo iba a sacar, porque en $4^{\circ}$ de la E.S.O nada más lo podemos aprobar limpio, no nos puede quedar ninguna, y me dijo que lo iba a llevar arrastrando y ya más por mi madre, mi madre quiere que siga jugando, lo único que quería es que le sacara los estudios, pero yo como no sabía si de verdad me lo iba a poder sacar o no pues lo deje. (Entrevista a PI. 46)

P.- $\mathrm{Y}$ en ese momento que decidiste dejarlo ¿alguien de tu alrededor tu madre, tu familia te animaron a continuar?

RU.- No, todo lo contrario, mi madre estaba hasta las narices, ella fue la que me metió la idea en la cabeza, me decía lo tienes que dejar, lo tienes que dejar, que así no puedes seguir, que el año que viene ya te cuenta la nota, el año que viene tu no vas a poder estar así, y de hecho este año me quería apuntar pero no para competir

Pensar a Prática, Goiânia, v. 14, n. 2, p. 1-15, maio/ago. 2011 
sino para hacer algo de deporte, porque llevo dos años sin hacer nada y no me dejó apuntarme. (Entrevista a RU. 92-93)

A pesar de toda la presión por abandonar el estudio, esta acción directa de la familia no tiene que concebirse únicamente ligada a una acción autoritaria. Depende del estilo educativo que exista en cada ámbito familiar. Así nos podemos encontrar otros estilos como el democrático, que hace que la decisión de abandono sea en mayor medida asumida por la propia joven y comprenda la repercusión de su elección.

P.-¿Recibiste ayuda, de tu familia, de tus amigos?¿Que tipo de ayuda recibiste en ese momento cuando tu decidiste dejarlo?

CR.- Pues por parte de mis padres, ellos lo dejaron mas bien a mi elección, ellos decían que si yo podía seguir con las dos cosas pues que siguiese, pero que si me sentía muy agobiada que a lo mejor lo dejase un tiempo y cuando ya pudiese yo seguir que lo intentase otra vez, pero que lo mirase yo como estaba yo mejor (...) (Entrevista a CR. 70-71)

En este planteamiento se deja en manos de las hijas la decisión final, aunque se orienta según un esquema de valores que posee la familia. No se impone sino que se dialoga y discute razonadamente. Es una carga para la propia joven esa decisión, pero va adquiriendo hábitos de autonomía y responsabilidad. Es evidente que por acción directa de la familia muchas de estas chicas han decidido que era preferible el estudio (algo que ocurre con todos los casos anteriores, ya sea un estilo educativo u otro), pero la acción puede ser también indirecta: no se obliga o aconseja a dejar el estudio pero la persona se socializa en un ambiente en el que hay unas prioridades en los valores. Se observa fundamentalmente esta acción indirecta de los padres y las madres en aquellas personas que poseen un mayor sentido de futuro, puesto que se plantean la necesidad del abandono por una serie de principios propios que son el resultado del proceso de socialización. Este sentido sólo es posible compararlo con las chicas que están inmersas en un estilo familiar democrático. Pero la causa, aunque sea indirecta, es la misma, es decir, una influencia del entorno familiar para dejar el deporte a favor del estudio.

P.- Entonces por parte de tu familia ¿te echaron una mano?

EV.- Sí, mis padres decían que si yo necesitaba tiempo para estudiar o para lo que fuera, que no pasaba nada, pero que siempre viene bien hacer deporte para estar en forma, que tienes una actividad que hacer, no estar todo el día metido en tu casa.

P.- ¿Y entonces al final la decisión la tomaste tu sola? EV.- Sí. (Entrevista a EV. 110-115)

Las adolescentes se han socializado en un ambiente en el que se han adquirido una serie de principios. Con ello, la propia persona hace propios esos valores y los utiliza sin presiones familiares. Así, las hijas han adquirido los valores de su entorno, unos valores en los que han priorizado ciertos aspectos sobre otros.

Pensar a Prática, Goiânia, v. 14, n. 2, p. 1-15, maio/ago. 2011 
El ambiente deportivo: Interacción entre personas.

La interacción que se produce en el deporte está dirigida en dos sentidos: con las compañeras (si es un deporte colectivo) y con el entrenador o la entrenadora (tanto en un deporte individual como colectivo). Ya se ha expuesto que en deportes individuales, y en chicas con éxito escolar, las causas por las que lo han abandonado están motivadas por una interferencia con los estudios, pero hay que añadir que los problemas en la interacción con otras personas pueden servir de apoyo a esa decisión.

Hay aspectos negativos a destacar, como son las relaciones con el entrenador/a. En los deportes individuales los conflictos suelen venir desde la propia figura del entrenador/a, como alguien autoritario al que hay que respetar y acostumbrarse:

R.- ¿Que tal tu relación con la entrenadora?.

RU.- Bien, es una persona un poquito así, o sea es simpática y eso, pero que también tiene una forma de ser un poquito seca, la tienes que saber tratar, al principio cuando era pequeña la tenía miedo. Luego, cuando la conoces ya lo que te dice te da lo mismo mas o menos, pero al principio... (Entrevista a RU 65-70)

También se muestra como las experiencias narradas de la Entrenadora, como deportista, influyen para la reflexión en la total dedicación al deporte, aunque el carácter agonista de la disciplina contribuye para intentar alcanzar la excelencia personal:

P.- ¿Tu no tenias tan claro que quisieras triunfar en tu deporte favorito?

RU.- No se, a lo mejor es que ... [la entrenadora], también como ella lo ha pasado muy mal, porque ella nos lo ha dicho muchas veces, que si no comía que si no se que, ella nunca nos ha obligado a no comer, a lo mejor te veía con un bollo de chocolate y te decía: "el chocolate que te engorda", pero no te obligaba a no comer, porque luego vas a competiciones y hay gente como en ... [distrito] que el club de allí se lo toma muy en serio, demasiado, ves a las niñas pequeñas llorando porque la profesora le tiene puesta la pierna en el hombro y les duele y las chicas a todo llorar y las profesoras les da lo mismo, y eso ... [la entrenadora] nunca lo hubiera hecho .... Ella siempre ha dicho que para divertirnos, que a ella le exigieron demasiado y se lo paso muy mal y siempre nos ha dado el punto de vista de que el deporte a gran escala es muy duro y es muy malo, pues entonces... . Yo sí me hubiera metido más, no me hubiera importado. (Entrevista a RU. 106-111)

También en éste tipo de deportes se suele dar el caso de que sea el padre el que asume el papel de entrenador, con lo que la presión puede ser mucho más fuerte porque se incrementa el sentido de compromiso y deber que se proyecta desde las jugadoras a sus entrenadores; el miedo a desilusionar es mucho más fuerte, tanto que es la madre quien media entre la deportista y su entrenador: 
PI.- Es que a él [el padre-entrenador] le gusta, a mi me gustaba, pero yo veía que mi padre quería una campeona y yo tampoco quería pero al final si que me sentía bastante presionada. A mí no me apetecía nada, días que no te apetece jugar en invierno, lo que menos te apetece a las seis de la tarde es ponerte y ¡hala! a la calle, a jugar. (Entrevista a PI. 54-55)

En el caso de las chicas que participaban en un deporte colectivo, la interacción con otras compañeras es fundamental para crear un clima positivo en la práctica, puesto que influye en las actitudes hacia el deporte. En los casos de abandono, la interacción negativa con las compañeras ha sido una de las principales causas alegadas por las entrevistadas.

PI.- Yo pienso que lo importante es más participar que ganar, pero a ellas solo les importaba ganar. Y en ese momento salté yo y le dije:'Déjame, no se que", dice: "Bueno, como no te lo pares, verás", y salí y efectivamente me lo paré, entonces cogí la pelota y le dije: "¿Que no me lo podía parar?, ¿Tan poco valoráis a las jugadoras?, pues mira aquí os quedáis" y me fui.(Entrevista a PI. 44)

Como se observa, hay varios aspectos en la interacción que son influyentes. Entre ellos estaría este primero, que es percibir una falta de aprecio por parte de las demás. Si en un deporte colectivo no se recibe aquello que se espera por parte de los integrantes del grupo es difícil que exista un acuerdo voluntario de permanecer juntas. El lazo entre los integrantes del equipo en ese deporte debe ser lo suficientemente fuerte para que un deporte colectivo pueda ser practicado.

P.- ¿Y por qué no elegiste otra actividad y elegiste el fútbol?, ¿Por eso?.

PI.- Si por eso, porque es que yo tampoco pensaba meterme en un equipo pero me lo comentaron y yo vi que se lo pasaban muy bien y que era una relación y que podías conocer a muchas personas, no se, sí me gustaba. (Entrevista a PI. 36-39)

P.- ¿Y por qué elegiste el voleibol?

TA.- No sé, porque me gusta, me gusta muchísimo ese deporte, veo que hay compenetración de equipo, no sé, me gusta bastante. (Entrevista a TA. 51-58)

Si se examinan el conjunto de las manifestaciones realizadas por las adolescentes, se observa que las causas deportivas, por sí solas, no son determinantes para el abandono de la práctica. Sí pueden llegar a influir conjuntamente con otros aspectos, pero no actúan en solitario. Este párrafo lo plantea claramente: 
CR.-[ ...] porque si en el colegio te mandan unas cosas no lo vas a dejar lo que vas hacer el día de mañana por la gimnasia, porque la gimnasia sabes que son hasta unos ciertos años, luego como no te dediques a ser entrenadora otra cosa no vas a hacer, porque tu sabes que hasta los 20 años y ya allí no vas a hacer nada. (Entrevista a CR. 61)

En este caso es una exigencia en el deporte el tener cierta edad, pero eso no justifica que se haya dejado incluso antes. Es un discurso que lo utiliza para dar sentido a una decisión que ha venido provocada por la presión en los estudios. Una presión que ha obligado a decidir, siguiendo su esquema de valores. El acusar a los aspectos deportivos del abandono es una forma de poder argumentar una decisión que, planteada de otro modo, no sería tan satisfactoria: el estudio es mejor que la gimnasia (una elección decidida según sus esquemas de prioridades).

Este discurso es utilizado con bastante frecuencia, culpabilizando a los aspectos deportivos por una decisión que ha sido obligada por otras circunstancias. Ninguna hubiera dejado un deporte que les gustaba por exigencias deportivas, han tenido que concurrir ciertos determinantes para que provoquen la decisión final.

P.- Y, ¿por qué lo dejaste?.

PA.- Pues ..., porque como siempre ha dicho mi entrenador, del atletismo comen dos ¿no?, entonces a mi me gusta y he competido en mi comunidad y hombre, me he quedado tercera, pero yo tengo claro que no voy a llegar a ser una estrella del atletismo nunca ¿no? (Entrevista a PA. 43-44)

Parece por tanto que las adolescentes asumen la existencia de exigencias deportivas, en general la actividad abandonada era un deporte que les gustaba realizar, pero se ha producido una situación que ha imposibilitado la práctica. Puede ser que sea necesaria una determinada edad, que sea muy dura la práctica, que se requiera un gran entrenamiento, que no sea la mejor... pero todo ello es posible asumirlo por algo que les gusta realizar. Es determinante que hayan concurrido otros factores adicionales para haber cesado su participación.

\section{Conclusiones y consideraciones finales}

A tenor de los resultados obtenidos, la respuesta a la pregunta de ¿por qué se pierde la vinculación con la práctica sistemática del deporte y de la actividad física en chicas jóvenes que han construido gran parte de sus vivencias dentro de un marco favorable? no permite ofrecer una respuesta única que cierre plenamente esta cuestión. Por otra parte, la metodología cualitativa seguida, posibilita aportar una visión, desde la reflexión interpretativa, de las diferentes caras de una realidad compleja.

Al analizar las causas del abandono, aparece sistemáticamente el "tiempo" como factor determinante. Pero un análisis en mayor profundidad muestra un sumatorio de motivos que conducen a esa percepción de no tener tiempo para lo importante. Lo importante, para la mayoría de las adolescentes entrevistadas, se centra en su actividad 
10.5216/rpp.v14i2.12932

académica, que al final se identifica como lo verdaderamente relevante de cara al futuro. ¿Por qué las chicas conceden tanta importancia, y en su opinión bastante más que los chicos, a su formación y resultados académicos?. ¿Podemos estar asistiendo a una lucha por superar estereotipos culturales que sólo otorgan plena independencia a las mujeres que son capaces de alcanzar un estatus social relevante a través de su desarrollo profesional?. Cabe preguntarse si el rendimiento académico cobraría un papel tan relevante si los sujetos de la investigación hubieran pertenecido al género masculino.

Las interacciones sociales, como base de la continuidad o retirada del deporte, ocupan un espacio importante. Tanto en el plano familiar como en el relacional, las presiones sufridas desde estos espacios han jugado un papel preponderante en las adolescentes entrevistadas a la hora de decidir abandonar la práctica deportiva. Unas veces de forma sutil u otras desde la imposición, aparece la figura de una madre o de un padre que a pesar de apoyar plenamente la práctica de actividad física, cuando llega el momento de la incertidumbre ante las presiones del contexto escolar optan por provocar en las jóvenes la conveniencia de reutilizar el tiempo de práctica deportiva hacia espacios que socialmente ofrecen sobre unas perspectivas de rentabilidad mayor que las aportadas por la actividad física.

El malestar de la convivencia deportiva, en el ámbito relacional dentro del microclima deportivo en el que se encuentran, constituye un factor que incita a la retirada: la presión se manifiesta cuando aparece la disensión y el malestar con las compañeras o el entrenador o entrenadora. El conflicto aflora y la solución por la que se opta es la del abandono.

En las adolescentes entrevistadas, la ruptura con la actividad física y deportiva ha sido prácticamente total. Parece claro que el abandono de la práctica deportiva está aparejada con una etapa de crisis que podría catalogarse de superación. Paralelamente, es en esta fase donde puede influir más el aspecto educativo del deporte, cuando se enfrentan a la superación del proceso que suele conllevar el abandono.

Como consideración final, indicar la necesidad de fortalecer los hábitos y vínculos con la actividad física y el deporte que mantienen las chicas en edades tempranas y que progresivamente van abandonando. Para ello, el conjunto de factores de influencia negativa identificados en este estudio pueden integrarse en futuras iniciativas y acciones, atendiendo especialmente a la familia y el grupo de amigos/as, al ambiente del grupo deportivo y la figura del profesor/a o entrenador/a. El centro escolar, paralelamente, surge como el elemento básico, de gran importancia, en la creación de ofertas y posibilidades de práctica de deportes y actividades físicas, coincidentes con las necesidades e intereses de las adolescentes, que manteniendo un carácter de participación abierta, ofrezca alternativas que posibiliten a las chicas mantener su implicación y compromiso con el deporte.

\section{Agradecimientos}

Este trabajo ha sido subvencionado por el Consejo Superior de Deportes dentro de sus Ayudas para Proyectos de Investigación en Ciencias del Deporte. Agradecer también al Dpto. de Didáctica de la Expresión Corporal de la Universidad de Granada su colaboración para el análisis de los datos.

Pensar a Prática, Goiânia, v. 14, n. 2, p. 1-15, maio/ago. 2011 
10.5216/rpp.v14i2.12932

\section{Referencias}

CORBIN, C. B.; PANGRAZI, R. P.; LeMASURIER G. C. Physical activity for children: Current patterns and guidelines. President's Council of Physical Fitness and Sport Research Digest, 5 (2), p. 1-8, 2004.

CURRIE, C.; ROBERTS, C.; MORGAN, A.; SMITH, R.; SETTERTOBULTE, W.; SAMDAL, O.; RASMUSEN, V. B. Young people's health in context. Health Behavior in School-aged Children (HBSC) study: international report from the 2001/2002 survey. Health Policy for Children and Adolescents (HEPCA) Series $\mathrm{n}^{\circ} 4$. Copenhagen: World Health Organization Regional Office for Europe, 2004.

CURRIE, C. ; GABHAINN, S. N. ; GODEAU, E. ; ROBERTS, C.; SMITH, R.; CURRIE, D.; PICKET, W.; RICHTER, M.; MORGAN, A.; BARNEKOW, V. Inequalities in Young People's Health. Health Behavior in School-aged Children (HBSC) study: international report from the 2005/2006 survey. Copenhagen: World Health Organization Regional Office for Europe, 2008.

DWYER, J.M. ; ALLISON, K. R. ; GOLDENBERG, E.R.; FEIN, A. J.; YOSHIDA, K. K.; BOUTLIER, M. A. Adolescents girls' perceived barriers to participation in physical activity. Adolescence, 41 (161), p.75-89, 2006.

FERNÁNDEZ, E.; VÁZQUEZ, B.; CAMACHO, M.J.; SÁNCHEZ, F.; MARTÍNEZ DE QUEL, O.; RODRÍGUEZ, I.; RUBIA, A.; AZNAR, S. (2006). La inclusión de la actividad física y el deporte en el estilo de vida de las mujeres adolescentes: Estudio de los factores clave y pautas de intervención. Revista IcD. Estudios sobre Ciencias del Deporte. Serie de Investigación, 46, p. 19-63, 2006.

GOULD, D. Understanding Attrition in Children's Sport. In: D. GOULD, D.; WEISS, M. R. (Eds.), Advances in Pediatric Sport Sciences. Vol II. Behavioural issues. Champaign, Ill.: Human Kinetics, 1987, p. 61-85.

GOULD, D. Understanding Attrition in Children's Sport. In: SMITH, D.; BAR-ELI, M. (Eds.), Essential Reading in Sport and Exercise. Champaign, Ill.: Human Kinetics, 2007, p. 401-411.

RODRÍGUEZ, A.: Estudio de las causas de abandono de la práctica deportiva habitual en la población de 14, 15 y 16 años. Tesis Doctoral no publicada. Granada: Universidad de Granada. 1998.

RODRÍGUEZ, G., GIL, J., GARCÍA, E.; ETXEBERRÍA, J. Análisis de datos cualitativos asistido por ordenador: AQUAD y NUDIST. Barcelona: PPU, 1995.

SAARINEN, P. Not all students take an interests ins sports, en Proceedings of the $7^{\text {th }}$ Congress of the European Federation of Sport Psychology, Leipzig: Deutsche Hochschule für Korperkultur, 1987, p. 563-566. 
SERRA, J. R. Estudio epidemiológico de los niveles de actividad física en los estudiantes de Educación Secundaria Obligatoria. Apunts: Educación Física y Deportes, 83, p. 25 34, 2006.

SLATER, A.; TIGGEMAN, M. "Uncool to do sport": A focus group Study od adolescent girls' reasons for withdrawing from physical activity. Psychology of Sport and Exercise, 11, p. 619-626, 2010.

SLEAP, M.; WORMALD, H. Perceptions of Physical Activity among Young Women aged 16 and 17 years. European Journal Of Physical Education, 6, p. 26-37, 2001.

TELAMA, R.; NAUl, R.; NUPPONEN, H.; RYCHTECKY, A.; VUOLlE, P. Physical fitness, sporting lifestyles, and Olympic ideals:cross-cultural studies on youth sport in Europe. Sport science studies; vol. 11. Schorndorf: Verlag Karl Hofmann, 2002.

WHITE, A.; COAKLEY, J.: Making decisions: The response of young people in the Medway towns to the 'Ever Thought of Sport?' campaign, London: Greater London and South East Region Sports Council, 1986.

WHITEHEAD, S.; BIDDLE, S. Adolescent girls' perceptions of physical activity: A focus group study. European Physical Education Review, 14 (2), p. 243- 262, 2008.

\section{ENTENDIMENTO DO ABANDONO DO ESPORTE DE MENINAS ADOLESCENTES.}

Resumo: O objetivo deste estudo é analisar o abandono do esporte de meninas adolescentes, suas experiências e identificar os fatores que contribuíram para a sua retirada dessa prática. O projeto é etnográfica e interpretativa, por meio de entrevistas retrospectivas com os adolescentes (14-17 anos) que abandonaram o esporte recentemente. A análise qualitativa dos dados foi realizada com o programa NUD*IST Vivo versão 6.0. Os resultados mostram a interação de um conjunto de fatores de influência entre os quais a dedicação interações acadêmicas e sociais, que são configurados como uma base de continuidade, especialmente a influência da família e das interacções pessoais como determinantes do ambiente esportivo.

Palavras-chave: Abandono. Esporte. Atividade Física. Meninas Adolescentes.

\section{UNDERSTANDING THE SPORT WITHDRAWAL OF ADOLESCENT GIRLS}

Abstract: The purpose of this paper is to analyse the sport withdrawal adolescent girls, their experiences and identifying the factors that have contributed to the leaving of the practice. The design is ethnographic and interpretive, using retrospective interviews with teenage girls (14-17 years) who have left the sport recently. The qualitative data analysis was performed with the program Nud*ist Vivo version 6.0. The results show the interaction of a set of influencing factors among which it has to be pointed the dedication 
10.5216/rpp.v14i2.12932

academic and social interactions, which are configured as a basis of continuity, especially the influence of family and personal interactions as determinants of the sports environment.

Keywords: Withdrawal. Sport. Physical Activity. Teenage Girls.

Endereço para correspondência:

efgarcia@edu.ucm.es

Emilia Fernández García

Universidad Complutense de Madrid

Ciudad Universitaria - 28040 Madrid

Pensar a Prática, Goiânia, v. 14, n. 2, p. 1-15, maio/ago. 2011 